\title{
Assessment of Cadmium Toxicity in Drinking Water of Eight Talukas of Upper Sindh
}

\author{
Noor Ul Hassan Shar* and Ghulam Qadir Shar \\ Institute of Chemistry, Shah Abdul Latif University, Khairpur, Sindh, Pakistan. \\ *Corresponding Author Email:noorulhassan4228@gmail.com \\ Received 21October 2021, Revised 09December 2021, Accepted 17 December 2021
}

\begin{abstract}
In the present study, 240 groundwater samples were collected from eight Talukas of upper Sindh. The cadmium (Cd) content was determined by atomic absorption spectrophotometer. Concentration $(\mu \mathrm{g} / \mathrm{L})$, Daily Intake of Metals (DIM), Health Risk Indexes (HRI) for children and adults caused due to $\mathrm{Cd}$ were measured through equations of EPA and WHO. Mean concentration of Cd in different Talukas of upper Sindh was found as; Daharki $(6.20 \mu \mathrm{g} / \mathrm{L})$, Ubauro $(7.73 \mu \mathrm{g} / \mathrm{L})$, Kashmore $(7.53 \mu \mathrm{g} / \mathrm{L})$, Tangwani $(7.73 \mu \mathrm{g} / \mathrm{L})$, Garhi Khairo $(6.20 \mu \mathrm{g} / \mathrm{L})$, Thul $(5.00 \mu \mathrm{g} / \mathrm{L})$, Qambar $(7.674 \mu \mathrm{g} / \mathrm{L})$ and Miro Khan $(7.47 \mu \mathrm{g} / \mathrm{L})$. Moreover, percent contamination of each Taluka was found as, Daharki (80\%), Ubauro (93\%), Kashmore (93\%), Tangwani (93\%), Gharhi Khairo (70\%), Thul (56\%), Qambar (93\%) and Miro Khan (93\%). The DIM of Cd for adult and children was calculated the range for adults was found as; Daharki (0.06 - 0.36), Ubauro (0.06 $0.42)$, Kashmore (0.06 - 0.36), Tangwani (0.06 - 0.42), Garhi Khairo (0.03 - 0.56), Thul (0.03 0.56), Qambar (0.08 - 0.39) and Miro Khan (0.08 - 0.39) $\mathrm{mg} / \mathrm{kg}-\mathrm{d}$, whereas, for children DIM of Cd was found lower than adults. The HRI values show that children compared to adults are under the potential risk threat due to HRI values $>1$. Therefore, it can be suggested that groundwater found in above stated areas is unsafe for drinking purposes and must be treated before consumption.
\end{abstract}

Keywords: Cadmium, Upper Sindh, Talukas, Groundwater, atomic absorption spectrophotometer

\section{Introduction}

Drinking water must be safe from contaminants because it is life; therefore, we must be anxious about its safety. Close connection has been observed between the geologic environment and chronic diseases. The geochemical atmosphere is responsible for the casual aspect of severe health issues [1].Various researchers have conducted studies to interpret the relation between potable water, geochemical environment, and diseases among humans [2]. Integral parts of suspended sediments or dissolved substances are considered the primary source of water elements. Nevertheless, dissolved materials in streams or rivers have the maximum potential of causing the most harmful effects. Consequently, groundwater contamination may be caused when elements stored in sediments of the riverbed percolate into the subterranean water. The contamination level of groundwater depends on the closeness of the well to the geological source $[3,4]$. Evaluation of potable water's physical and chemical properties is critical for determining that the water is safe for drinking and cooking purposes [5]. Diseases and health problems may be caused due to Inorganic elements and pathogens [6]. Cadmium $(\mathrm{Cd})$ is a trace and toxic element and may be found in petroleum, coal, and rocks. No evidence for the 
essentiality of $\mathrm{Cd}$ is indicated to humans. Groundwater and surface water may be contaminated with $\mathrm{Cd}$ by geologic deposits when in contact with water [7]. The $\mathrm{Cd}$ is highly toxic, damages kidneys and heart, and may cause cancer. Low intake of $\mathrm{Cd}$ may cause vomiting, nausea, headache, and cough, whereas its higher dose may cause liver, kidney failure, human hypertension, renal failure, and bone disorder [8]. Potable water contaminated with $\mathrm{Cd}$ may cause chronic anemia by prolonged exposure. When it is induced to activity by $\mathrm{Cd}$, it gets bound to zinc and copper [9].

Chronic exposure to $\mathrm{Cd}$ is responsible for a broad range of chronic and acute effects in humans. Accumulation of $\mathrm{Cd}$ in the human body occurs in kidneys, causing renal tubular damage, which is an important health consequence. Development of kidney stones, hypercalciuria, and disturbance in calcium metabolism are also effects of $\mathrm{Cd}$ exposure. Poor water quality that may produce $\mathrm{Cd}$ poisonous effect has produced an international dilemma [10]. Besides $\mathrm{Cr}, \mathrm{Hg}, \mathrm{Pb}$, and $\mathrm{As}$, no physiological function of $\mathrm{Cd}$ is found and is believed to be a toxin [11]. During lactation, $\mathrm{Cd}$ eliminates from the body slowly through milk, saliva, urine, and kidneys. Various adverse effects of $\mathrm{Cd}$ are resulted due to $\mathrm{Cd}$ exposure in humans, like harm to the hemopoietic, adrenal structure, osteomalacia, testicular damage, pulmonary edema, and hepatic and renal dysfunction [12]. The sight, hearing weakness, damage of skeletal, liver, cardiovascular, and kidneys systems may occur due to low levels of Cd [13]. The main purpose of the current study was to evaluate the concentration of $\mathrm{Cd}$ and its human health risk assessment, calculate the Daily Intake of $\mathrm{Cd}$ and determine the toxic level of $\mathrm{Cd}$ in the drinking water of upper Sindh.

\section{Materials and Methods Study Area}

The weather condition of upper Sindh is excessively warm in summer and modest in winter (Fig. 1). The maximum and minimum temperatures noted are $52.8{ }^{\circ} \mathrm{C}$ and $-3.9{ }^{\circ} \mathrm{C}$, respectively. The mean rainfall per annum in this area is $122.5 \mathrm{~mm}$, usually in the monsoon season from July to September.

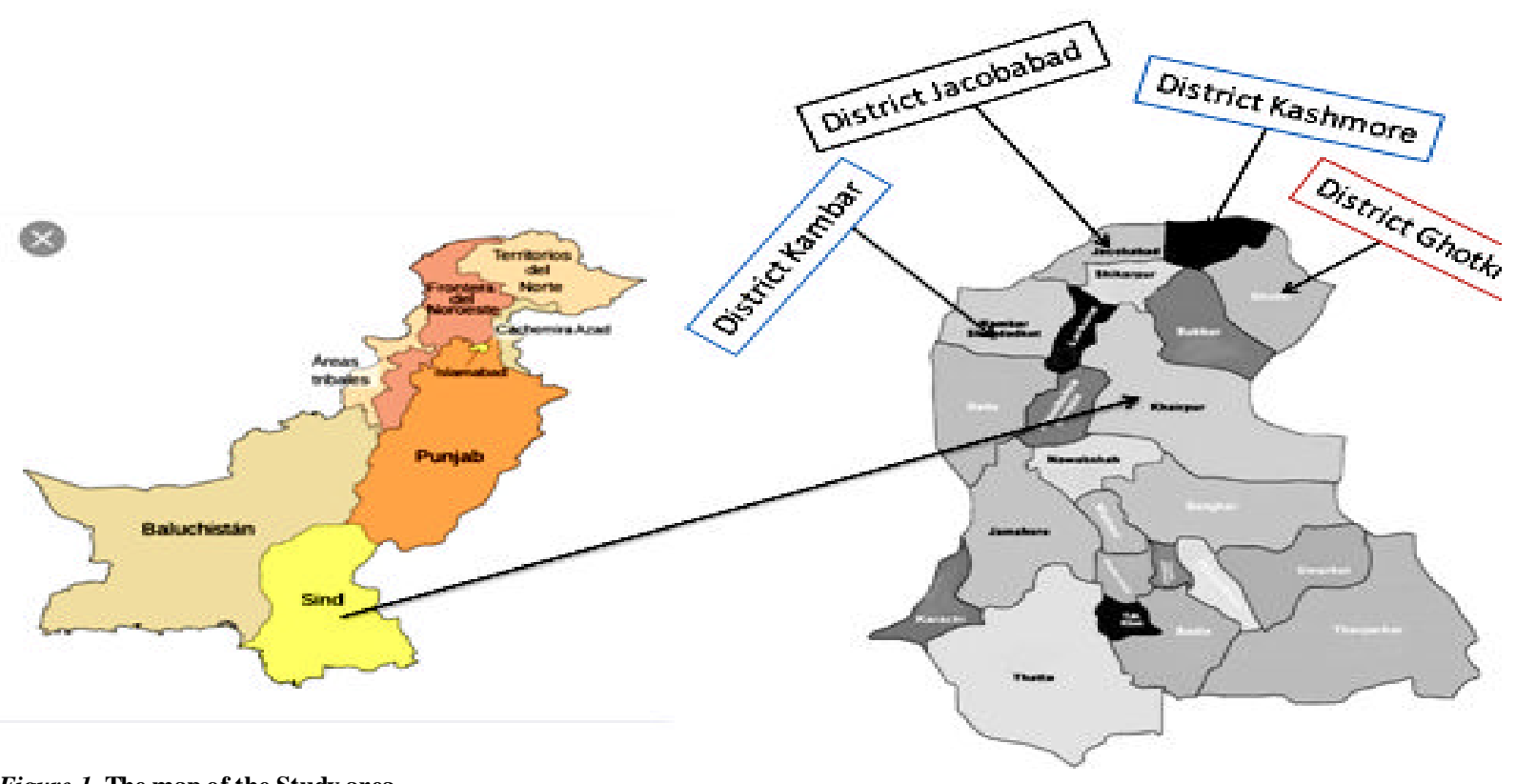

Figure 1. The map of the Study area 
The air is generally dry because the thermal equator passes from the upper Sindh. The area of upper Sindh is 6,790 square kilometers and is located at $27^{\circ} 56^{\prime}$ and $28^{\circ}$ $27^{\prime}$ N. and $68^{\circ}$, and $69^{\circ} 44^{\prime}$ E. Upper Sindh has the Thar Desert on the eastern side, Suleman Range in North, Bahurai range in the west and Khirthar range in Southwest. The massive Indus river flows from north to south at the south-eastern side of Jacobabad; moreover, so many canals and streams consisting of the Bolan river are present on the western and northern sides. The flow of nonperennial streams is always found towards Sindh since the south western, western, and northern parts belong to the Baluchistan plateau [14].

\section{Chemicals and Reagents}

Reagents used to prepared a standard solution of $\mathrm{Cd}$ were bought from Merck (Darmstadt, Germany), and de-ionized water was used to prepare the required strength for $\mathrm{Cd}$ analysis.

\section{Sampling}

To get groundwater samples, it is necessary to run hand pumps for at least five minutes to eliminate insoluble impurities and sand particles andbring depth water of required elemental amounts [15]. Water used for drinking purposes was obtained from hand pumps whose depth was $30-70$ feet. Plastic bottles were used to collect samples of 1500 $\mathrm{mL}$ of water. Eight Talukas of upper Sindh were selected to get water samples from various hand pumps to draw water from the ground with a depth of $30-70$ feet. The global positioning system method was used to collect water samples from upper Sindh. In total, 240 potable water samples were collected from eight Talukas of upper Sindh. Collected water samples were acidified with 1 $\mathrm{mL}$ of $\mathrm{HNO}_{3}$ at the sampling location. Samples were transferred and placed in the dark at $4^{\circ} \mathrm{C}$ for $\mathrm{Cd}$ analysis [16].

\section{Analytical Procedures}

Atomic Absorption Spectrophotometer (Analytic Jena) was used to analyze the concentration of $\mathrm{Cd}$ in water samples under standard operating conditions in Pakistan Council of Research in Water Resources Islamabad, Ministry of Science and Technology, Islamabad. Triplicate water samples were analyzed for data quality assurance. Three standards of $1.25 \mathrm{mg} / \mathrm{L}, 2.5$ $\mathrm{mg} / \mathrm{L}$, and $5.0 \mathrm{mg} / \mathrm{L}$ were analyzed after every ten samples to check the results of the instrument. At a confidence level of $95 \%$, the reproducibility of results was observed. Thus, for results, average interpretation values were utilized. All glassware was washed with $2 \%$ $\mathrm{HNO}_{3}$, and the chemicals used in this work were of analytical grade [17].

\section{Health Risk Assessment for Human}

Health risk assessment for humans by using contaminated drinking water by $\mathrm{Cd}$ was assessed through formulas for health risks such as Daily Intake of Metals (DIM) and Health Risk Indexes (HRI).

\section{Daily Intake of Metals (DIM)}

Oral ingestion plays a critical role in $\mathrm{Cd}$ intake in humans. However, there are different pathways through which heavy metals may be contacted to the human body, for example, oral intake, inhalation, food chain, and dermal interaction [18]. The following equation was used to calculate the DIM adapted from

$\mathrm{DIM}=\frac{\mathrm{Cm} \times \mathrm{DIW}}{\mathrm{Bw}}$

Where $C m, D l w$ and $B W$ are the concentration of heavy metals in water, average daily intake of water ( $2 \mathrm{~L}$ per day for adults and $1 \mathrm{~L}$ per day for children) [19], and average body weight (72 and $23.7 \mathrm{~kg}$ for adults and children, respectively) [19]. 
Health Risk Indexes (HRIs) of Heavy Metals

Formula (ii) given below was used to calculate HRIs of toxic metals by ingestion of water [20].

$\mathrm{HTI}=\frac{\mathrm{DIM}}{\mathrm{RfD}}$

Where DIM and RfD are the average daily intake and toxicity oral reference dose of heavy metals. The $R f D$ value of $\mathrm{Cd}$ is given as $0.5 \mu \mathrm{g} / \mathrm{kg}$-day. The level of $\mathrm{Cd}$ is considered safe when HRIs are less than 1 [21].

\section{Results and Discussion Cadmium Contamination}

Cd has no known physiological function in mammals and is one of the most lethal metals for human beings. Its exposure to humans may occur through water, food, and cigarettes inhalation. Once absorbed in the human body, $\mathrm{Cd}$ retains strongly all over life, having a half-life of 25 to 30 years [22].There are many industrial uses of $\mathrm{Cd}$, such as plastics, batteries, coatings, alloys, pigments, and paints. For the production of alkaline batteries, most of the $\mathrm{Cd}$ is used as an electrode. $\mathrm{Cd}$ elimination occurs from industrial processes as well as from $\mathrm{Cd}$ smelters into groundwater, fertilizers, and sewage sludge which may stay in sediments and soils for various years and be absorbed by plants [22]. Thus, noteworthy contact of Cd to humans may be absorbed by polluted goods, particularly vegetables, fruits, grains, cereals, and polluted beverages. Moreover, $\mathrm{Cd}$ may be inhaled via municipal waste burning [10].

Mean concentration of $\mathrm{Cd}$ in different Talukas of upper Sindh was found as; Daharki $(6.20 \mu \mathrm{g} / \mathrm{L})$, Ubauro $(7.73 \mu \mathrm{g} / \mathrm{L})$, Kashmore $(7.53 \mu \mathrm{g} / \mathrm{L})$, Tangwani $(7.73 \mu \mathrm{g} / \mathrm{L})$, Garhi Khairo $(6.20 \mu \mathrm{g} / \mathrm{L})$, Thul $5.0 \mu \mathrm{g} / \mathrm{L})$, Qambar $(7.674 \mu \mathrm{g} / \mathrm{L})$ and Miro Khan $(7.47 \mu \mathrm{g} / \mathrm{L})$. Moreover, percent contamination of each
Taluka was found as, Daharki (80\%), Ubauro (93\%), Kashmore (93\%), Tangwani (93\%), Gharhi Khairo (70\%), Thul (56\%), Qambar (93\%) and Miro Khan (93\%). It can be observed from the mean results of $\mathrm{Cd}$ that water samples of all eight Talukas of Upper Sindh are found contaminated compared with the WHO limit of $3 \mu \mathrm{g} / \mathrm{L}$ (Table 1). Cd concentration in water of the different parts of the world and various parts of Pakistan can be seen in Table 2. Maximum $\mathrm{Cd}$ content of $170.0 \mu \mathrm{g} / \mathrm{L}$ was found in the water of Indonesia [30], while according to literature,the water of Germany displayed a minimum of $4.8 \mu \mathrm{g} / \mathrm{L}$ [35]. The highest $\mathrm{Cd}$ content of $251 \mu \mathrm{g} / \mathrm{L}$ was determined from Chiniot, Pakistan [37], while the lowest of 1.4 $\mu \mathrm{g} / \mathrm{L}$ was observed from Multan [36].

Table 1. Concentration $(\mu \mathrm{g} / \mathrm{L})$ of cadmium in drinking water of upper Sindh.

\begin{tabular}{|c|c|c|c|c|c|c|c|c|}
\hline $\begin{array}{c}\text { Sample } \\
\text { No. }\end{array}$ & DK & UB & $\mathbf{K M}$ & TW & GK & TH & QB & MK \\
\hline 1 & 4 & 4 & 4 & 13 & 3 & 4 & 3 & 3 \\
\hline 2 & 4 & 4 & 12 & 11 & 8 & 9 & 4 & 4 \\
\hline 3 & 4 & 7 & 4 & 5 & 6 & 4 & 6 & 6 \\
\hline 4 & 2 & 7 & 8 & 7 & 8 & 3 & 5 & 5 \\
\hline 5 & 3 & 12 & 13 & 12 & 8 & 8 & 3 & 3 \\
\hline 6 & 2 & 7 & 8 & 4 & 7 & 5 & 4 & 4 \\
\hline 7 & 2 & 9 & 8 & 4 & 6 & 9 & 14 & 14 \\
\hline 8 & 9 & 12 & 4 & 7 & 20 & 20 & 4 & 4 \\
\hline 9 & 6 & 14 & 2 & 14 & 8 & 7 & 14 & 14 \\
\hline 10 & 6 & 15 & 9 & 15 & 4 & 6 & 13 & 13 \\
\hline 11 & 6 & 13 & 6 & 13 & 9 & 9 & 9 & 9 \\
\hline 12 & 7 & 13 & 5 & 5 & 8 & 8 & 7 & 7 \\
\hline 13 & 7 & 6 & 8 & 9 & 9 & 4 & 4 & 4 \\
\hline 14 & 3 & 7 & 2 & 7 & 3 & 3 & 7 & 7 \\
\hline 15 & 11 & 7 & 4 & 3 & 1 & 1 & 11 & 11 \\
\hline 16 & 13 & 7 & 11 & 7 & 4 & 4 & 6 & 6 \\
\hline 17 & 11 & 7 & 7 & 7 & 4 & 2 & 7 & 7 \\
\hline 18 & 6 & 6 & 5 & 6 & 3 & 3 & 9 & 9 \\
\hline 19 & 4 & 2 & 13 & 2 & 9 & 1 & 5 & 5 \\
\hline 20 & 4 & 6 & 9 & 13 & 2 & 2 & 4 & 4 \\
\hline 21 & 6 & 9 & 11 & 6 & 3 & 5 & 4 & 4 \\
\hline 22 & 3 & 7 & 13 & 7 & 3 & 3 & 13 & 13 \\
\hline 23 & 4 & 3 & 9 & 7 & 7 & 2 & 8 & 8 \\
\hline 24 & 11 & 4 & 8 & 4 & 8 & 8 & 9 & 9 \\
\hline 25 & 8 & 5 & 9 & 5 & 9 & 2 & 8 & 8 \\
\hline 26 & 7 & 5 & 6 & 5 & 3 & 3 & 7 & 7 \\
\hline 27 & 7 & 6 & 6 & 6 & 9 & 9 & 8 & 8 \\
\hline 28 & 8 & 11 & 8 & 7 & 1 & 1 & 13 & 13 \\
\hline 29 & 9 & 12 & 7 & 9 & 8 & 4 & 11 & 11 \\
\hline 30 & 9 & 5 & 7 & 12 & 5 & 1 & 4 & 4 \\
\hline Min & 2 & 2 & 2 & 2 & 1 & 1 & 3 & 3 \\
\hline Max & 13 & 15 & 13 & 15 & 20 & 20 & 14 & 14 \\
\hline Mean & 6.2 & 7.7 & 7.5 & 7.7 & 6.2 & 5.0 & 7.5 & 7.5 \\
\hline SD & 3.0 & 3.5 & 3.1 & 3.6 & 3.7 & 3.9 & 3.5 & 3.5 \\
\hline
\end{tabular}


Table 2. Cadmium Content $(\mu \mathrm{g} / \mathrm{L})$ in various parts of world and Pakistan.

\begin{tabular}{llllll}
\hline \multicolumn{2}{c}{$\begin{array}{c}\text { Different } \\
\text { Countries }\end{array}$} & Ref & \multicolumn{2}{c}{$\begin{array}{c}\text { Different } \\
\text { parts of Pakistan }\end{array}$} & Ref \\
\hline India & 60.0 & {$[23]$} & Ghorabari & 80.0 & {$[32]$} \\
Iran & 8.36 & {$[24]$} & Mardan & 80.0 & {$[33]$} \\
Germany & 4.80 & {$[25]$} & Vehari & 10.0 & {$[34]$} \\
Algeria & 32.80 & {$[26]$} & Khane wal & 200.0 & {$[35]$} \\
Lebanon & 81.32 & {$[27]$} & Multan & 1.40 & {$[36]$} \\
India & 10.0 & {$[28]$} & Chiniot & 251.0 & {$[37]$} \\
Bangladesh & 7.0 & {$[29]$} & Northern Sindh & 10.90 & {$[38]$} \\
Indonesia & 170.0 & {$[30]$} & Sijawal Junejo & 25.90 & {$[39]$} \\
Malaysia & 3.43 & {$[31]$} & Rato Dero & 13.70 & {$[40]$} \\
\hline
\end{tabular}

\section{Daily Intake (DIM) of Cadmium}

The Daily Intake of $\mathrm{Cd}$ in groundwater of study area for adults was found from all of the eight Talukas as, Daharki (0.06 - 0.36 $\mu \mathrm{g} / \mathrm{kg}$-day), Ubauro (0.06 - $0.42 \mu \mathrm{g} / \mathrm{kg}$-day), Kashmore $(0.06-0.36 \mu \mathrm{g} / \mathrm{kg}$-day $)$, Tangwani (0.06 - $0.42 \mu \mathrm{g} / \mathrm{kg}$-day), Garhi Khairo (0.03 $0.56 \mu \mathrm{g} / \mathrm{kg}$-day $)$, Thul (0.03 - $0.56 \mu \mathrm{g} / \mathrm{kg}$-day), Qambar (0.08 - $0.39 \mu \mathrm{g} / \mathrm{kg}$-day) and Miro
Khan (0.08 - $0.39 \mu \mathrm{g} / \mathrm{kg}$-day) (Table 3). The average daily intake of $\mathrm{Cd}$ in groundwater of upper Sindh was found as, 0.17, 0.21, 0.21, $0.21,0.17,0.14,0.21$ and $0.21 \mu \mathrm{g} / \mathrm{kg}$-day from Daharki, Ubauro, Kashmore, Tangwani, Garhi Khairo, Thul, Qambar and Miro Khan, respectively (Table 3). While for children daily intake of $\mathrm{Cd}$ was observed as, Daharki (0.08 - $0.55 \mu \mathrm{g} / \mathrm{kg}$-day), Ubauro (0.08 - 0.63 $\mu \mathrm{g} / \mathrm{kg}$-day), Kashmore $(0.08-0.55 \mu \mathrm{g} / \mathrm{kg}$ day), Tangwani (0.08 - $0.63 \mu \mathrm{g} / \mathrm{kg}$-day), Garhi Khairo (0.04 - $0.84 \mu \mathrm{g} / \mathrm{kg}$-day), Thul (0.04 $0.84 \mu \mathrm{g} / \mathrm{kg}$-day), Qambar (0.13 - $0.59 \mu \mathrm{g} / \mathrm{kg}$ day) and Miro Khan (0.13 - $0.59 \mu \mathrm{g} / \mathrm{kg}$-day). The average daily intake of $\mathrm{Cd}$ for children from upper Sindh was as under, 0.26, 0.33, $0.32,0.33,0.26,0.21,0.32$ and $0.32 \mu \mathrm{g} / \mathrm{kg}$ day in Talukas Daharki, Ubauro, Kashmore, Tangwani, Garhi Khairo, Thul, Qambar and Miro Khan, respectively (Table 4).

Table 3. Daily intake of cadmium ( $\mu \mathrm{g} / \mathrm{kg}$-day) for adul ts in the drinking of upper Sindh.

\begin{tabular}{|c|c|c|c|c|c|c|c|c|}
\hline Sample No. & DK & UB & $\mathbf{K M}$ & TW & GK & TH & QB & MK \\
\hline 1 & 0.11 & 0.11 & 0.11 & 0.36 & 0.08 & 0.11 & 0.08 & 0.08 \\
\hline 2 & 0.11 & 0.11 & 0.33 & 0.31 & 0.22 & 0.25 & 0.11 & 0.11 \\
\hline 3 & 0.11 & 0.19 & 0.11 & 0.14 & 0.17 & 0.11 & 0.17 & 0.17 \\
\hline 4 & 0.06 & 0.19 & 0.22 & 0.19 & 0.22 & 0.08 & 0.14 & 0.14 \\
\hline 5 & 0.08 & 0.33 & 0.36 & 0.33 & 0.22 & 0.22 & 0.08 & 0.08 \\
\hline 6 & 0.06 & 0.19 & 0.22 & 0.11 & 0.19 & 0.14 & 0.11 & 0.11 \\
\hline 7 & 0.06 & 0.25 & 0.22 & 0.11 & 0.17 & 0.25 & 0.39 & 0.39 \\
\hline 8 & 0.25 & 0.33 & 0.11 & 0.19 & 0.56 & 0.56 & 0.11 & 0.11 \\
\hline 9 & 0.17 & 0.39 & 0.06 & 0.39 & 0.22 & 0.19 & 0.39 & 0.39 \\
\hline 10 & 0.17 & 0.42 & 0.25 & 0.42 & 0.11 & 0.17 & 0.36 & 0.36 \\
\hline 11 & 0.17 & 0.36 & 0.17 & 0.36 & 0.25 & 0.25 & 0.25 & 0.25 \\
\hline 12 & 0.19 & 0.36 & 0.14 & 0.14 & 0.22 & 0.22 & 0.19 & 0.19 \\
\hline 13 & 0.19 & 0.17 & 0.22 & 0.25 & 0.25 & 0.11 & 0.11 & 0.11 \\
\hline 14 & 0.08 & 0.19 & 0.06 & 0.19 & 0.08 & 0.08 & 0.19 & 0.19 \\
\hline 15 & 0.31 & 0.19 & 0.11 & 0.08 & 0.03 & 0.03 & 0.31 & 0.31 \\
\hline 16 & 0.36 & 0.19 & 0.31 & 0.19 & 0.11 & 0.11 & 0.17 & 0.17 \\
\hline 17 & 0.31 & 0.19 & 0.19 & 0.19 & 0.11 & 0.06 & 0.19 & 0.19 \\
\hline 18 & 0.17 & 0.17 & 0.14 & 0.17 & 0.08 & 0.08 & 0.25 & 0.25 \\
\hline 19 & 0.11 & 0.06 & 0.36 & 0.06 & 0.25 & 0.03 & 0.14 & 0.14 \\
\hline 20 & 0.11 & 0.17 & 0.25 & 0.36 & 0.06 & 0.06 & 0.11 & 0.11 \\
\hline 21 & 0.17 & 0.25 & 0.31 & 0.17 & 0.08 & 0.14 & 0.11 & 0.11 \\
\hline 22 & 0.08 & 0.19 & 0.36 & 0.19 & 0.08 & 0.08 & 0.36 & 0.36 \\
\hline 23 & 0.11 & 0.08 & 0.25 & 0.19 & 0.19 & 0.06 & 0.22 & 0.22 \\
\hline 24 & 0.31 & 0.11 & 0.22 & 0.11 & 0.22 & 0.22 & 0.25 & 0.25 \\
\hline 25 & 0.22 & 0.14 & 0.25 & 0.14 & 0.25 & 0.06 & 0.22 & 0.22 \\
\hline 26 & 0.19 & 0.14 & 0.17 & 0.14 & 0.08 & 0.08 & 0.19 & 0.19 \\
\hline 27 & 0.19 & 0.17 & 0.17 & 0.17 & 0.25 & 0.25 & 0.22 & 0.22 \\
\hline 28 & 0.22 & 0.31 & 0.22 & 0.19 & 0.03 & 0.03 & 0.36 & 0.36 \\
\hline 29 & 0.25 & 0.33 & 0.19 & 0.25 & 0.22 & 0.11 & 0.31 & 0.31 \\
\hline 30 & 0.25 & 0.14 & 0.19 & 0.33 & 0.14 & 0.03 & 0.11 & 0.11 \\
\hline Min: & 0.06 & 0.06 & 0.06 & 0.06 & 0.03 & 0.03 & 0.08 & 0.08 \\
\hline Max: & 0.36 & 0.42 & 0.36 & 0.42 & 0.56 & 0.56 & 0.39 & 0.39 \\
\hline Mean & 0.17 & 0.21 & 0.21 & 0.21 & 0.17 & 0.14 & 0.21 & 0.21 \\
\hline SD & 0.08 & 0.10 & 0.09 & 0.10 & 0.10 & 0.11 & 0.10 & 0.10 \\
\hline
\end{tabular}


Table 4. Daily intake of cadmium ( $\mu \mathrm{g} / \mathrm{kg}$-day) for children in the drinking of upper Sindh.

\begin{tabular}{|c|c|c|c|c|c|c|c|c|}
\hline Sample No. & DK & UB & $\mathbf{K M}$ & TW & GK & TH & QB & MK \\
\hline 1 & 0.17 & 0.17 & 0.17 & 0.55 & 0.13 & 0.17 & 0.13 & 0.13 \\
\hline 2 & 0.17 & 0.17 & 0.51 & 0.46 & 0.34 & 0.38 & 0.17 & 0.17 \\
\hline 3 & 0.17 & 0.30 & 0.17 & 0.21 & 0.25 & 0.17 & 0.25 & 0.25 \\
\hline 4 & 0.08 & 0.30 & 0.34 & 0.30 & 0.34 & 0.13 & 0.21 & 0.21 \\
\hline 5 & 0.13 & 0.51 & 0.55 & 0.51 & 0.34 & 0.34 & 0.13 & 0.13 \\
\hline 6 & 0.08 & 0.30 & 0.34 & 0.17 & 0.30 & 0.21 & 0.17 & 0.17 \\
\hline 7 & 0.08 & 0.38 & 0.34 & 0.17 & 0.25 & 0.38 & 0.59 & 0.59 \\
\hline 8 & 0.38 & 0.51 & 0.17 & 0.30 & 0.84 & 0.84 & 0.17 & 0.17 \\
\hline 9 & 0.25 & 0.59 & 0.08 & 0.59 & 0.34 & 0.30 & 0.59 & 0.59 \\
\hline 10 & 0.25 & 0.63 & 0.38 & 0.63 & 0.17 & 0.25 & 0.55 & 0.55 \\
\hline 11 & 0.25 & 0.55 & 0.25 & 0.55 & 0.38 & 0.38 & 0.38 & 0.38 \\
\hline 12 & 0.30 & 0.55 & 0.21 & 0.21 & 0.34 & 0.34 & 0.30 & 0.30 \\
\hline 13 & 0.30 & 0.25 & 0.34 & 0.38 & 0.38 & 0.17 & 0.17 & 0.17 \\
\hline 14 & 0.13 & 0.30 & 0.08 & 0.30 & 0.13 & 0.13 & 0.30 & 0.30 \\
\hline 15 & 0.46 & 0.30 & 0.17 & 0.13 & 0.04 & 0.04 & 0.46 & 0.46 \\
\hline 16 & 0.55 & 0.30 & 0.46 & 0.30 & 0.17 & 0.17 & 0.25 & 0.25 \\
\hline 17 & 0.46 & 0.30 & 0.30 & 0.30 & 0.17 & 0.08 & 0.30 & 0.30 \\
\hline 18 & 0.25 & 0.25 & 0.21 & 0.25 & 0.13 & 0.13 & 0.38 & 0.38 \\
\hline 19 & 0.17 & 0.08 & 0.55 & 0.08 & 0.38 & 0.04 & 0.21 & 0.21 \\
\hline 20 & 0.17 & 0.25 & 0.38 & 0.55 & 0.08 & 0.08 & 0.17 & 0.17 \\
\hline 21 & 0.25 & 0.38 & 0.46 & 0.25 & 0.13 & 0.21 & 0.17 & 0.17 \\
\hline 22 & 0.13 & 0.30 & 0.55 & 0.30 & 0.13 & 0.13 & 0.55 & 0.55 \\
\hline 23 & 0.17 & 0.13 & 0.38 & 0.30 & 0.30 & 0.08 & 0.34 & 0.34 \\
\hline 24 & 0.46 & 0.17 & 0.34 & 0.17 & 0.34 & 0.34 & 0.38 & 0.38 \\
\hline 25 & 0.34 & 0.21 & 0.38 & 0.21 & 0.38 & 0.08 & 0.34 & 0.34 \\
\hline 26 & 0.30 & 0.21 & 0.25 & 0.21 & 0.13 & 0.13 & 0.30 & 0.30 \\
\hline 27 & 0.30 & 0.25 & 0.25 & 0.25 & 0.38 & 0.38 & 0.34 & 0.34 \\
\hline 28 & 0.34 & 0.46 & 0.34 & 0.30 & 0.04 & 0.04 & 0.55 & 0.55 \\
\hline 29 & 0.38 & 0.51 & 0.30 & 0.38 & 0.34 & 0.17 & 0.46 & 0.46 \\
\hline 30 & 0.38 & 0.21 & 0.30 & 0.51 & 0.21 & 0.04 & 0.17 & 0.17 \\
\hline Min: & 0.08 & 0.08 & 0.08 & 0.08 & 0.04 & 0.04 & 0.13 & 0.13 \\
\hline Max: & 0.55 & 0.63 & 0.55 & 0.63 & 0.84 & 0.84 & 0.59 & 0.59 \\
\hline Mean & 0.26 & 0.33 & 0.32 & 0.33 & 0.26 & 0.21 & 0.32 & 0.32 \\
\hline SD & 0.13 & 0.15 & 0.13 & 0.15 & 0.16 & 0.17 & 0.15 & 0.15 \\
\hline
\end{tabular}


Health Risk Indexes (HRIs) of Cadmium

The range of HRI values of $\mathrm{Cd}$ in drinking water for adults in various Talukas of upper Sindh under study were observed as, Daharki (0.1 - 0.7), Ubauro (0.1 - 0.8), Kashmore $(0.1-0.7)$, Tangwani $(0.1-0.8)$, Garhi Khairo (0.2 - 1.1), Thul (0.1 - 1.1), Qambar (0.2 - 0.8) and Miro Khan (0.2 - 0.8). Two Talukas Garhi Khairo and Thul of upper Sindh showed HRI value of $\mathrm{Cd}>1$ in groundwater. All the other Talukas understudy declared the HRI value of $\mathrm{Cd}$ within the safe limit (Table 5).
The HRI of Cd for children in groundwater of different Talukas of upper Sindh was found as, Daharki (0.2 - 1.1), Ubauro (0.2 - 1.3), Kashmore (0.2 - 1.1), Tangwani (0.2 - 1.3), Garhi Khairo (0.1 - 1.7), Thul (0.08 - 1.7), Qambar (0.3 - 1.2) and Miro Khan (0.1 - 1.0). The mean value of HRI of $\mathrm{Cd}$ in groundwater of various Talukas of upper Sindh was observed as, Daharki (0.52), Ubauro (0.65), Kashmore (0.64), Tangwani (0.65), Garhi Khairo (0.52), Thul (0.42), Qambar (0.63) and Miro Khan (0.52) (Table. 5).

Table 5. Heal th riskindex of cadmium in drinking water of upper Sindh.

\begin{tabular}{|c|c|c|c|c|c|c|c|c|c|c|c|c|c|c|c|c|}
\hline \multirow{2}{*}{$\begin{array}{c}\text { Sample } \\
\text { No. }\end{array}$} & \multicolumn{8}{|c|}{ Adults } & \multicolumn{8}{|c|}{ Children } \\
\hline & $D K$ & $\overline{U B}$ & $K M$ & $T W$ & $G K$ & $\mathrm{TH}$ & $Q B$ & MK & $D K$ & $U B$ & $K M$ & $T W$ & $G K$ & $T H$ & $Q B$ & $M K$ \\
\hline 1 & 0.2 & 0.2 & 0.2 & 0.7 & 0.2 & 0.2 & 0.2 & 0.7 & 0.3 & 0.3 & 0.3 & 1.1 & 0.3 & 0.3 & 0.3 & 1.0 \\
\hline 2 & 0.2 & 0.2 & 0.7 & 0.6 & 0.4 & 0.5 & 0.2 & 0.6 & 0.3 & 0.3 & 1 & 0.9 & 0.7 & 0.8 & 0.3 & 0.9 \\
\hline 3 & 0.2 & 0.4 & 0.2 & 0.3 & 0.3 & 0.2 & 0.3 & 0.2 & 0.3 & 0.6 & 0.3 & 0.4 & 0.5 & 0.3 & 0.5 & 0.3 \\
\hline 4 & 0.1 & 0.4 & 0.4 & 0.4 & 0.4 & 0.2 & 0.3 & 0.2 & 0.2 & 0.6 & 0.7 & 0.6 & 0.7 & 0.3 & 0.4 & 0.3 \\
\hline 5 & 0.2 & 0.7 & 0.7 & 0.7 & 0.4 & 0.4 & 0.2 & 0.6 & 0.3 & 1 & 1.1 & 1 & 0.7 & 0.7 & 0.3 & 0.9 \\
\hline 6 & 0.1 & 0.4 & 0.4 & 0.2 & 0.4 & 0.3 & 0.2 & 0.2 & 0.2 & 0.6 & 0.7 & 0.3 & 0.6 & 0.4 & 0.3 & 0.3 \\
\hline 7 & 0.1 & 0.5 & 0.4 & 0.2 & 0.3 & 0.5 & 0.8 & 0.2 & 0.2 & 0.8 & 0.7 & 0.3 & 0.5 & 0.8 & 1.2 & 0.3 \\
\hline 8 & 0.5 & 0.7 & 0.2 & 0.4 & 1.1 & 1.1 & 0.2 & 0.2 & 0.8 & 1 & 0.3 & 0.6 & 1.7 & 1.7 & 0.3 & 0.3 \\
\hline 9 & 0.3 & 0.8 & 0.1 & 0.8 & 0.4 & 0.4 & 0.8 & 0.3 & 0.5 & 1.2 & 0.2 & 1.2 & 0.7 & 0.6 & 1.2 & 0.4 \\
\hline 10 & 0.3 & 0.8 & 0.5 & 0.8 & 0.2 & 0.3 & 0.7 & 0.6 & 0.5 & 1.3 & 0.8 & 1.3 & 0.3 & 0.5 & 1.1 & 0.8 \\
\hline 11 & 0.3 & 0.7 & 0.3 & 0.7 & 0.5 & 0.5 & 0.5 & 0.3 & 0.5 & 1.1 & 0.5 & 1.1 & 0.8 & 0.8 & 0.8 & 0.4 \\
\hline 12 & 0.4 & 0.7 & 0.3 & 0.3 & 0.4 & 0.4 & 0.4 & 0.4 & 0.6 & 1.1 & 0.4 & 0.4 & 0.7 & 0.7 & 0.6 & 0.7 \\
\hline 13 & 0.4 & 0.3 & 0.4 & 0.5 & 0.5 & 0.2 & 0.2 & 0.3 & 0.6 & 0.5 & 0.7 & 0.8 & 0.8 & 0.3 & 0.3 & 0.5 \\
\hline 14 & 0.2 & 0.4 & 0.1 & 0.4 & 0.2 & 0.2 & 0.4 & 0.1 & 0.3 & 0.6 & 0.2 & 0.6 & 0.3 & 0.3 & 0.6 & 0.2 \\
\hline 15 & 0.6 & 0.4 & 0.2 & 0.2 & 0.1 & 0.1 & 0.6 & 0.2 & 0.9 & 0.6 & 0.3 & 0.3 & 0.1 & 0.1 & 0.9 & 0.3 \\
\hline 16 & 0.7 & 0.4 & 0.6 & 0.4 & 0.2 & 0.2 & 0.3 & 0.4 & 1.1 & 0.6 & 0.9 & 0.6 & 0.3 & 0.3 & 0.5 & 0.6 \\
\hline 17 & 0.6 & 0.4 & 0.4 & 0.4 & 0.2 & 0.1 & 0.4 & 0.7 & 0.9 & 0.6 & 0.6 & 0.6 & 0.3 & 0.2 & 0.6 & 1 \\
\hline 18 & 0.3 & 0.3 & 0.3 & 0.3 & 0.2 & 0.2 & 0.5 & 0.4 & 0.5 & 0.5 & 0.4 & 0.5 & 0.3 & 0.3 & 0.8 & 0.6 \\
\hline 19 & 0.2 & 0.1 & 0.7 & 0.1 & 0.5 & 0.1 & 0.3 & 0.4 & 0.3 & 0.2 & 1.1 & 0.2 & 0.8 & 0.1 & 0.4 & 0.6 \\
\hline 20 & 0.2 & 0.3 & 0.5 & 0.7 & 0.1 & 0.1 & 0.2 & 0.1 & 0.3 & 0.5 & 0.8 & 1.1 & 0.2 & 0.2 & 0.3 & 0.1 \\
\hline 21 & 0.3 & 0.5 & 0.6 & 0.3 & 0.2 & 0.3 & 0.2 & 0.2 & 0.5 & 0.8 & 0.9 & 0.5 & 0.3 & 0.4 & 0.3 & 0.3 \\
\hline 22 & 0.2 & 0.4 & 0.7 & 0.4 & 0.2 & 0.2 & 0.7 & 0.6 & 0.3 & 0.6 & 1.1 & 0.6 & 0.3 & 0.3 & 1.1 & 0.8 \\
\hline 23 & 0.2 & 0.2 & 0.5 & 0.4 & 0.4 & 0.1 & 0.4 & 0.3 & 0.3 & 0.3 & 0.8 & 0.6 & 0.6 & 0.2 & 0.7 & 0.5 \\
\hline 24 & 0.6 & 0.2 & 0.4 & 0.2 & 0.4 & 0.4 & 0.5 & 0.5 & 0.9 & 0.3 & 0.7 & 0.3 & 0.7 & 0.7 & 0.8 & 0.8 \\
\hline 25 & 0.4 & 0.3 & 0.5 & 0.3 & 0.5 & 0.1 & 0.4 & 0.4 & 0.7 & 0.4 & 0.8 & 0.4 & 0.8 & 0.2 & 0.7 & 0.6 \\
\hline 26 & 0.4 & 0.3 & 0.3 & 0.3 & 0.2 & 0.2 & 0.4 & 0.2 & 0.6 & 0.4 & 0.5 & 0.4 & 0.3 & 0.3 & 0.6 & 0.3 \\
\hline 27 & 0.4 & 0.3 & 0.3 & 0.3 & 0.5 & 0.5 & 0.4 & 0.1 & 0.6 & 0.5 & 0.5 & 0.5 & 0.8 & 0.8 & 0.7 & 0.2 \\
\hline 28 & 0.4 & 0.6 & 0.4 & 0.4 & 0.1 & 0.1 & 0.7 & 0.5 & 0.7 & 0.9 & 0.7 & 0.6 & 0.1 & 0.1 & 1.1 & 0.8 \\
\hline 29 & 0.5 & 0.7 & 0.4 & 0.5 & 0.4 & 0.2 & 0.6 & 0.3 & 0.8 & 1 & 0.6 & 0.8 & 0.7 & 0.3 & 0.9 & 0.4 \\
\hline 30 & 0.5 & 0.3 & 0.4 & 0.7 & 0.3 & 0.1 & 0.2 & 0.2 & 0.8 & 0.4 & 0.6 & 1 & 0.4 & 0.1 & 0.3 & 0.3 \\
\hline Min: & 0.1 & 0.1 & 0.1 & 0.1 & 0.1 & 0.1 & 0.2 & 0.1 & 0.2 & 0.2 & 0.2 & 0.2 & 0.1 & 0.1 & 0.3 & 0.1 \\
\hline Max & 0.7 & 0.8 & 0.7 & 0.8 & 1.1 & 1.1 & 0.8 & 0.7 & 1.1 & 1.3 & 1.1 & 1.3 & 1.7 & 1.7 & 1.2 & 1.0 \\
\hline Mean & 0.33 & 0.43 & 0.40 & 0.43 & 0.34 & 0.28 & 0.41 & 0.35 & 0.53 & 0.65 & 0.64 & 0.65 & 0.54 & 0.44 & 0.63 & 0.52 \\
\hline SD & 0.16 & 0.20 & 0.17 & 0.20 & 0.19 & 0.21 & 0.19 & 0.18 & 0.25 & 0.30 & 0.27 & 0.31 & 0.32 & 0.33 & 0.30 & 0.26 \\
\hline
\end{tabular}


Table 6. Correlation of Cadmium among potable water of upper Sindh.

\begin{tabular}{|c|c|c|c|c|c|c|c|c|}
\hline Talukas & Daharki & Ubauro & Kashmore & Tangwani & Garhi Khairo & Thul & Qambar & Miro Khan \\
\hline Daharki & 1 & & & & & & & \\
\hline Ubauro & .065 & 1 & & & & & & \\
\hline Kashmore & -.157 & -.221 & 1 & & & & & \\
\hline Tangwani & -.111 & $0.401^{*}$ & -.034 & 1 & & & & \\
\hline Garhi Khairo & .024 & 0.182 & -.049 & -.032 & 1 & & & \\
\hline Thul & .012 & $0.442^{*}$ & -.151 & .119 & $0.755^{* *}$ & 1 & & \\
\hline Qambar & .120 & $0.409^{*}$ & -.165 & -.034 & -.239 & -.068 & 1 & \\
\hline Miro Khan & .120 & $0.409^{*}$ & -.165 & -.034 & -.239 & -.068 & $1.000^{* *}$ & 1 \\
\hline
\end{tabular}

\section{Statistical Analysis}

Pearson correlation among potable water of eight Talukas of upper Sindh is shown in Table 6. A positive correlation was observed among Taluka Tangwani and Ubauro $\left(0.401^{*}\right)$. Cd in potable water of Taluka Thul and Ubauro also showed a positive correlation of $0.442^{*}$, whereas the Taluka Kambar and Ubauro also displayed a positive correlation of $0.409^{*}$. Taluka Thul and Garhi Khairo displayed a strong positive correlation of $0.755^{* *}$. The strongest positive correlation of $1.000^{* * *}$ was observed between Miro Khan and Qambar. All relations were observed significant at the level of 0.05 and 0.01 , respectively.

\section{Conclusion}

From the present study, it can be concluded that most of the potable water samples were contaminated due to higher $\mathrm{Cd}$ content. About $93 \%$ of water samples of Talukas Qambar, Miro Khan, Ubauro, Kashmore, and Tangwaniwere found contaminated. HRI values of most of the samples were found greater than one, which shows that the water of the study area is unsafe for the local population. HRI was found higher for children as compared toadults. The present work shows that groundwater in the study areas is hazardous for drinking purposes and must be treated before consumption. Therefore, this study suggests that the government should provide safe drinking water by installing reverse osmosis plants where people use unsafe water for drinking and cooking purposes.

\section{Conflict of Interest}

The authors declare that there is no conflict of interest.

\section{References}

1. C. M. D. Oliveira, Rev. Ambient. Água., 12 (2017) 985.

doi:10.4136/ambi-agua.2037

2. D. Karunanidhi, P. Aravinthasamy, T. Subramani and G. Muthusankar, Environ. Geochem. Health, 43 (2021) 931. https://doi.org/10.1007/s10653-02000613-3

3. A. Debnath, P. K. Singh and Y. C. Sharma, J. Environ. Manag., 298 (2021) 113378.

https://doi.org/10.1016/j.jenvman.2021.1 13378

4. M. A. Yusuf and T. A. Abiye, Groundwater Sustain. Develop., 9 (2019) 100222. https://doi.org/10.1016/i.gsd.2019.100222

5. U. Younas, S. Iqbal, A. Saleem, M. Iqbal, A. Nazir, S. Noureen, K. Mehmood and N. Nisar, Acta Ecologica Sinica, 37 (2017) 236. 
http://dx.doi.org/10.1016/j.chnaes.20 17.02 .002

6. E. Bloem, A. Albihn, J. Elving, L. Hermann, L. Lehmann, M. Sarvi, T. Schaaf, J. Schick, E. Turtola and K. Ylivainio, Sci. Total Environ., 607 (2017) 225.

https://www.elsevier.com/open-

access/userlicense/1.0/

7. F. A. Ababneh, Int. J. Anal. Chem., 2017 (2017) 1687

https://doi.org/10.1155/2017/6971916

8. G. Fatima, A. M. Raza, N. Hadi, N. Nigam and A. A. Mahdi, Indian J. Clin. Biochem., 34 (2019) 371.

https://doi.org/10.1007/s12291-01900839-8

9. O. B. Akpor, G. O. Ohiobor and D. T. Olaolu, Adv. Biosci. Bioeng., 2 (2014) 37.

https://doi:10.11648/j.ijepp.20140206.12

10. N. Idrees, B. Tabassum, E. F. Abd. Allah, A. Hashem, R. Sarah and M. Hashim. Saudi J. Biol. Sci., 25 (2018) 1365.

https://doi.org/10.1016/j.sjbs.2018.07.005

11. R. Abedi Sarvestani and M. Aghasi, Environ. Earth Sci., 78 (2019) 714. https://doi.org/10.1007/s12665-019$\underline{8723-0}$

12. G. Genchi, M. S. Sinicropi, G. Lauria, A. Carocci and A. Catalano, Int. J. Environ. Res. Public Health, 17 (2020) 3782. https://doi:10.3390/ijerph17113782

13. M. Mahurpawar, Int. J. Res. Granthaalayah, 3 (2015) 1.

https://doi.org/10.29121/granthaalayah.v 3.i9SE.2015.3282

14. S. Ali, Pak. J. Meteorol., 9 (2013) 59. http://www.pmd.gov.pk/rnd/rndweb/rnd new/journal.php\#18

15. A. R. Shar, G. Q. Shar, M. A. Jakhrani, S. A. Jakhrani, N. Zaman, N. U. H. Shar, M. B. Shar, A. H. Rind and K. A. Bhutto, Int. J. Biosci., 17 (2020) 287. http://dx.doi.org/10.12692/ijb/17.3.287-294

16. J. B. Fisher, K. P. Tu and D. D. Baldocchi, Remote Sens. Environ., 112 (2008) 901 . https://doi:10.1016/j.rse.2007.06.025

17. D. Guven and G. Akinci, Gazi Univ. J. Sci., 24 (2011) 29.

https://dergipark.org.tr/en/pub/gujs/issue /7418/96796

18. Z. I. Khan, K. Ahmad, S. Rehman, S. Siddique, H. Bashir, A. Zafar, M. Sohail, S. A. Ali, E. Cazzato and G. De Mastro, Environ. $\quad$ Sci. $\quad$ Poll. Res., 24 (2017) 947. doi:10.1007/s1 1356-016-7865-9

19. J. C. Egbueri, Groundwater Sustain. Develop., 10 (2020) 100292. https://doi.org/10.1016/j.gsd.2019.10029 $\underline{2}$

20. B. Chakraborty, S. Roy, A. Bera, P. P. Adhikary, B. Bera, D. Sengupta, G. S. Bhunia and P. K. Shit, Springer, Cham., 19 (2021) 327.

doi: 10.1007/978-3-030-63422-3_19

21. Rasool, S. Muhammad, M. Shafeeque, I. Ahmad, F. A. Al-Misned, H. A. ElSerehy, S. Ali, B. Murtaza and A. Sarwar, Human. Ecol. Risk. Assess.: An Int. J., 27 (2021) 16551670. https://doi.org/10.1080/10807039.2020.1 $\underline{870036}$

22. G. F. Nordberg, A. Bernard, G. L. Diamond, J. H. Duffus, P. Illing, M. Nordberg, I. A. Bergdahl, T. Jin and S. Skerfving, Pure Appl. Chem., 90 (2018) 755.

https://doi.org/10.1515/pac-2016-0910

23. R. Kaur, S. Sharma and H. Kaur. $J$. Pharmacol. Phytochem., SP1 (2019) 247.

https://www.phytojournal.com/archives/ 2019/vol8issue 1S/PartG/Sp-8-1-74571.pdf

24. N. Idrees, B. Tabassum, E. F. Abd. Allah, A. Hashem, R. Sarah and M. Hashim, Saudi J. Biol. Sci., 25 (2018) 
1365.

https://doi.org/10.1016/j.sjbs.2018.07.005

25. M. Qasemi, M. Shams, S.A. Sajjadi, M. Farhang, S. Erfanpoor, M. Yousefi, A. Zarei and M. Afsharnia, Biol. Trace Elem. Res., 192 (2019) 106.

https://doi.org/10.1007/s12011-019$\underline{1660-7}$

26. A. Kubier, K. Hamer and T. Pichler, Integ. Environ. Assess. Manag., 16 (2020) 103.

https://doi.org/10.1002/ieam.4198

27. N. Balli and E. Leghouchi, Glob. Nest. J., 20 (2018) 417.

https://journal.gnest.org/sites/default/file s/Submissions/gnest_02747/gnest_0274 7_published.pdf

28. D. A. Halwani, M. Jurdi, F. K. A. Salem, M. A. Jaffa, N. Amacha, R. R. Habib and H. R. Dhaini, Expos. Health, 12 (2020) 163.

https://doi.org/10.1007/s12403-019-

00301-3

29. K. Brindha, R. Paul, J. Walter, M. L. Tan and M. K. Singh, Environ. Geochem. Health, 42 (2020) 3819.

https://doi.org/10.1007/s10653-020$\underline{00637-9}$

30. M. A. T. Rahman, M. Paul, N. Bhoumik, M. Hassan, M. K. Alam and Z. Aktar, Appl. Water Sci., 10 (2020) 1. https://doi.org/10.1007/s13201-02001266-4

31. A. Tjahjono and D. Suwarno, J. Ecol. Eng., 19 (2018). 43 doi:10.12911/22998993/89715

32. M. F. Ahmed and M. B. Mokhtar, Int. J. Environ. Res. Public Health, 17 (2020) 2966. https://doi.org/10.3390/ijerph17082966

33. A. R. Shar, G. Q. Shar, S. M. Wassan, W. B. Jatoi, Z. Bhatti, M. A. Jakhrani and A. A. Shar, Pak. J. Anal. Environ. Chem., 22 (2021) 100. http://doi.org/10.21743/pjaec/2021.06.11
34. A. Rashid, M. Ayub, A. Javed, S. Khan, X. Gao, C. Li, Z. Ullah, T. Sardar, J. Muhammad and S. Nazneen, Geosci. Front., 12 (2021) 101128.

https://doi.org/10.1016/j.gsf.2020.12.009

35. S. Ahmad, M. Imran, B. Murtaza, M. Arshad, R. Nawaz, A. Waheed, H. M. Hammad, M. A. Naeem, M. Shahid and N. K. Niazi, Environ. Geochem. Health 43 (2021) 5195.

https://doi.org/10.1007/s10653-02100941-y

36. G. Murtaza, and H.R. Sabihakhurram, Int. J. Res. Stud. Sci. Eng. Technol., 5 (2018) 7. https://ijrsset.org/volume-5issue-11

37. Z. Iqbal, F. Abbas, A. Mahmood, M. Ibrahim, M. Gul, M. Yamin, B. Aslam, M. Imtiaz, N.N. Elahi, T.I. Qureshi and G.Z.H. Sial, Int. J. Environ. Sci. Technol., (2021) in press. https://doi.org/10.1007/s13762-02103611-9

38. S. Iram, R. Sultana, M. S. ud Din, M. N. Ahmad and Z. Shamrose, Int. J. Econ. Environ. Geol., 19 (2019) 21. https://doi.org/10.46660/ijeeg. Vol0.Iss0. 0.11

39. R. Shaikh, T. G. Kazi, H. I. Afridi, A. Akhtar, J. A. Baig and M. B. Arain, Environ. Pollut., 255 (219) 113305. https://doi.org/10.1016/j.envpol.2019.11 $\underline{3305}$

40. M. F. Lanjwani, M. Y. Khuhawar and T. M. J. Khuhawar, Appl. Water Sci., (2020) in press. https://doi.org/10.1007/s13201-019$\underline{1098-2}$ 